\title{
The oral microbiome - an update for oral healthcare professionals
}

\author{
M. Kilian, ${ }^{* 1}$ I. L. C. Chapple, ${ }^{2}$ M. Hannig, ${ }^{3}$ P. D. Marsh, ${ }^{4}$ V. Meuric, ${ }^{5}$ A. M. L. Pedersen, ${ }^{6}$ M. S. Tonetti, ${ }^{7}$ \\ W. G. Wade ${ }^{8}$ and E. Zaura ${ }^{9}$
}

\section{In brief}

Summarises the benefits of a healthpromoting oral microbiome for oral and general health.
Provides an overview of coevolution between humans and oral microbes.
Reviews factors associated with dysbiosis and implications for caries and periodontal disease.
Highlights existing strategies to preserve a balanced oral microbiome for practitioners and patients to follow.

For millions of years, our resident microbes have coevolved and coexisted with us in a mostly harmonious symbiotic relationship. We are not distinct entities from our microbiome, but together we form a 'superorganism' or holobiont, with the microbiome playing a significant role in our physiology and health. The mouth houses the second most diverse microbial community in the body, harbouring over 700 species of bacteria that colonise the hard surfaces of teeth and the soft tissues of the oral mucosa. Through recent advances in technology, we have started to unravel the complexities of the oral microbiome and gained new insights into its role during both health and disease. Perturbations of the oral microbiome through modern-day lifestyles can have detrimental consequences for our general and oral health. In dysbiosis, the finely-tuned equilibrium of the oral ecosystem is disrupted, allowing disease-promoting bacteria to manifest and cause conditions such as caries, gingivitis and periodontitis. For practitioners and patients alike, promoting a balanced microbiome is therefore important to effectively maintain or restore oral health. This article aims to give an update on our current knowledge of the oral microbiome in health and disease and to discuss implications for modern-day oral healthcare.

\section{Introduction}

Humans, like all complex multicellular eukaryotes, are not autonomous organisms, but biological units that include numerous microbial symbionts and their genomes. ${ }^{1}$ The microbes

'Department of Biomedicine, Faculty of Health, Aarhus University, Wilhelm Meyers allé 4, DK-8000, Aarhus C, Denmark; ${ }^{2} S$ chool of Dentistry, Institute of Clinical Sciences, College of Medical and Dental Sciences, University of Birmingham, Birmingham, UK; ${ }^{3}$ Clinic of Operative Dentistry, Periodontology and Preventive Dentistry, Saarland University, Homburg, Germany; ${ }^{4}$ School of Dentistry, University of Leeds, Leeds, UK; ${ }^{5}$ CHU Rennes, EA 1254 Microbiologie Risques infectieux, Université de Rennes 1, Rennes, France; ${ }^{6}$ Section of Oral Medicine, Clinical Oral Physiology, Oral

Pathology and Anatomy, Department of Odontology, Faculty of Health and Medical Sciences, University of Copenhagen, Copenhagen, Denmark; ${ }^{7}$ European Research Group on Periodontology, Genova, Italy and Department of Periodontology, The University of Hong Kong, Hong Kong, SAR China; ${ }^{8}$ Barts and The London School of Medicine and Dentistry, Queen Mary University of London, London, UK; ${ }^{9}$ Department of Preventive Dentistry, Academic Centre for Dentistry Amsterdam, University of Amsterdam and Vrije Universiteit Amsterdam, Gustav Mahlerlaan 3004, 1081 LA Amsterdam, the Netherlands

*Correspondence to: Mogens Kilian Email: kilian@biomed.au.dk

Refereed Paper. Accepted 25 October 2016 DOI: 10.1038/sj.bdj.2016.865

${ }^{\oplus B}$ British Dental Journal 2016; 221: 657-666 in and on our bodies form a functional organ that is fundamental to our health and physiology (Fig. 1). Together with our symbiotic microbial residents, we form a 'superorganism, or holobiont. The microbial component of the human holobiont is substantial, and at least equals the number of our own cells. ${ }^{2}$ The community of our microbial residents is referred to as our microbiome, a term that was coined by the Nobel prize laureate Joshua Lederberg to signify the ecological community of commensal, symbiotic, and pathogenic microorganisms that literally share our body space and have been all but ignored as determinants of health

Fig. 1 Positive effects of host-microbiome symbiosis

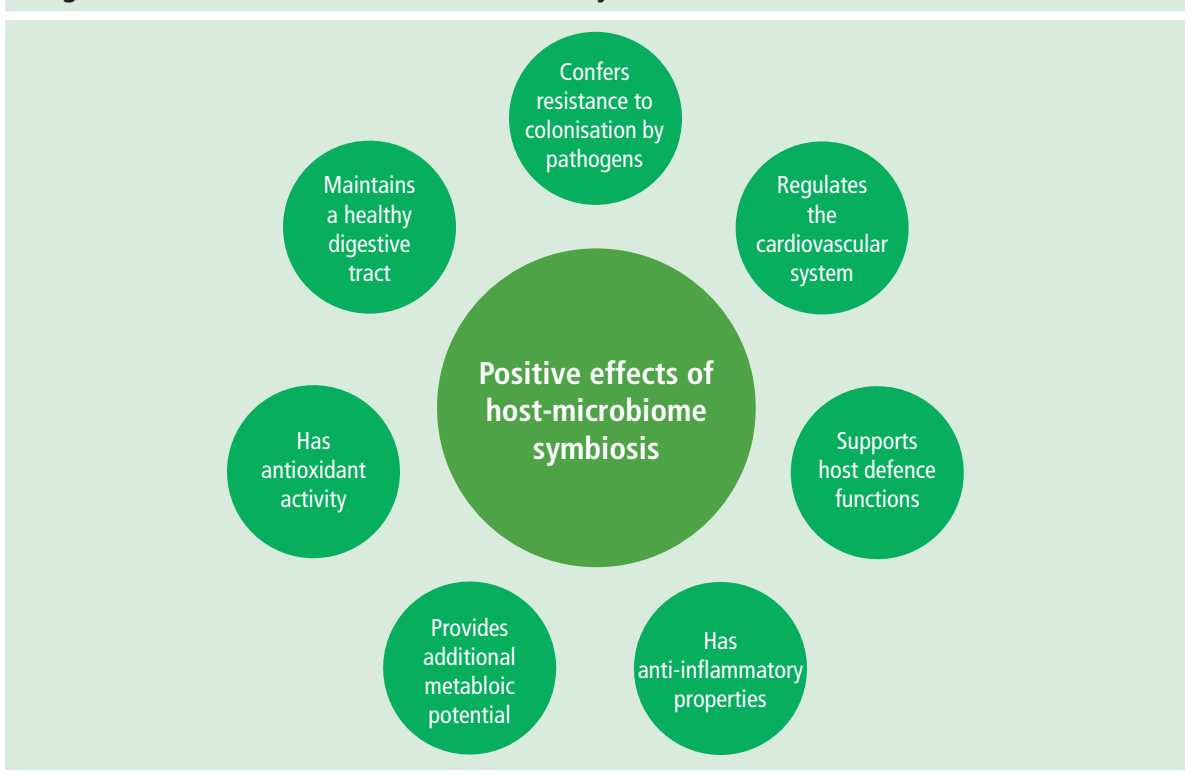




\begin{tabular}{|c|c|}
\hline Term & Definition \\
\hline 16S ribosomal RNA (16S rRNA) & $\begin{array}{l}\text { An RNA molecule (approximately } 1500 \text { nucleotides long) that is part of the small subunit of the ribosome in prokaryotes. } 16 \mathrm{~S} \text { rRNA } \\
\text { has both highly conserved regions, common to all prokaryotes, and hypervariable regions that are unique to particular species }\end{array}$ \\
\hline Biofilm & $\begin{array}{l}\text { A sessile community of microbes characterized by cells that are attached to a surface or to each other and embedded in a matrix } 0 \\
\text { extracellular polymeric substances }{ }^{20}\end{array}$ \\
\hline Bioinformatics & The application of computational techniques to analyse complex biological data such as genetic codes \\
\hline Coevolution & The parallel evolution of interacting species \\
\hline Community profiling & Characterisation of complex microbial communities by their $16 \mathrm{~S}$ rRNA gene sequences \\
\hline Dysbiosis & $\begin{array}{l}\text { A condition in which the normal microbiome population structure is disturbed, often through external burdens such as disease } \\
\text { states or medications }{ }^{7}\end{array}$ \\
\hline Epigenetics & The study of heritable changes in gene expression that are not caused by changes in DNA sequence $e^{95}$ \\
\hline Gene amplification & $\begin{array}{l}\text { An increase of gene copies by laboratory methods for research purposes. }{ }^{*} \text { Amplification of a gene produces enough copies of a } \\
\text { gene to allow for gene sequencing }\end{array}$ \\
\hline Gene sequencing & $\begin{array}{l}\text { A laboratory method to determine the precise order of nucleotides within a DNA molecule, enabling scientists to decipher genetic } \\
\text { information that is carried in a particular DNA segment (for example, the } 16 S \text { rRNA gene) }\end{array}$ \\
\hline Holobiont & The host organism and all its symbiotic microbial residents ${ }^{1}$ \\
\hline Metagenomics & $\begin{array}{l}\text { Analysis of the genetic information of a complex population, typically from microbes in an environmental or host sample. The } \\
\text { metagenome consists of the genomes of many individual microbes? }\end{array}$ \\
\hline Metatranscriptomics & Analysis of the active genes and species of the microbiome $e^{96}$ \\
\hline Microbiome & The sum of microbes, their genetic information, and the environment in which they interact ${ }^{7}$ \\
\hline Microbiota & All living microbial organisms constituting the microbiome ${ }^{7}$ \\
\hline Next-generation sequencing (NGS) & An umbrella term to describe a number of different modern high throughput sequencing technologies \\
\hline Operational taxonomic unit (OTU) & $\begin{array}{l}\text { The grouping of bacterial } 16 \mathrm{~S} \text { rRNA gene sequences by their similarity. }{ }^{7} \text { Sequences are typically grouped at a value between } 97 \% \\
\text { and } 99 \%\end{array}$ \\
\hline Phenotype & The observable physical characteristics of an organism, for example, appearance, behaviour or clinical presentation \\
\hline Phylogenetics & The study of the evolutionary relationships among groups of organisms \\
\hline Phylotype & A type of bacterium defined by its placement in a phylogenetic tree on the basis of its $16 \mathrm{~S}$ rRNA gene sequence \\
\hline Species & Coherent and distinct groups of bacteria that have been isolated, cultured and named ${ }^{*}$ \\
\hline Symbiosis & Two or more species living closely together in a long-term relationship' \\
\hline
\end{tabular}

and disease. ${ }^{3}$ The emergence of new genomic technologies, including next-generation sequencing (NGS) and bioinformatic tools, has provided a powerful means of understanding the contribution of the human microbiome to health. Ventures started in the last decade, such as the Human Microbiome Project ${ }^{4}$ and Metagenomics of the Human Intestinal Tract (MetaHIT), ${ }^{5}$ have generated the resources and expertise needed to characterise and understand the human microbiome. Our knowledge is set to grow further with future undertakings such as the recently announced National Microbiome Initiative, a partnership between federal US agencies, academia, charitable organisations and the private sector, who collectively have pledged over US\$ 500 million in research funding. ${ }^{6}$ We have learned that we are not colonised at random, but that our microbial residents have coevolved with us over millions of years. The relationship between microbiome and host is dynamic, and influenced by many aspects of modern lifestyle, such as diet, tobacco consumption and stress, which can alter our microbiome and its properties, and induce a state in which this finely tuned ecosystem is no longer in balance. To address this divergence and maintain a harmonious state to protect health and prevent disease, we must not focus on the host and its residents as separate units, but instead consider the holobiont as one.

The mouth is one of the most heavily colonised parts of our bodies. Several distinct habitats within the oral cavity support heterogeneous microbial communities that constitute an important link between oral and general health. The purpose of this article is to update oral healthcare practitioners on our current knowledge of the oral microbiome in health and disease, to review how molecular methods of microbial characterisation have advanced our understanding, and to discuss potential implications for clinical practice. For an introduction to key terms used throughout the text (highlighted in bold), please see the glossary in Table 1 .

\section{The oral microbiome: discussion}

\section{Our current understanding of} the biological evolution of the microbiome

There is evidence that resident microbes have been performing metabolic functions in animals for at least 500 million years. ${ }^{7}$ Coevolution is documented by the many similarities in the composition and organisation of the human microbiome to that of other mammals. ${ }^{8}$ The history of the relationship 


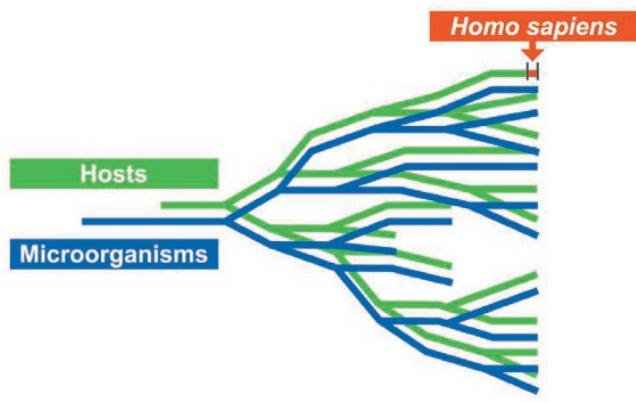

1.5 billion years

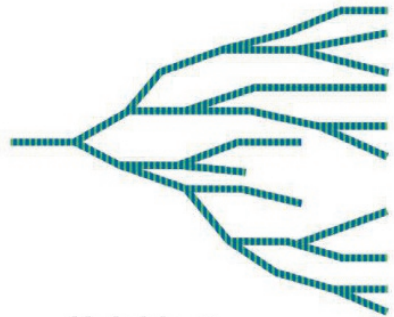

Holobiont

1.5 billion years

Fig. 2 History of the relationship between microorganisms and humans: The coevolution between microorganisms (blue lines) and their respective hosts (green lines) over a period of 1.5 billion years has resulted in mutual adaptation and functional integration as reflected in our own relationship to most of the microorganisms that colonise our body surfaces (Homo sapiens, red line)

between microorganisms and humans is outlined in Figure 2. In humans, coevolution has also resulted in minor, but important, differences between ethnic groups. ${ }^{9-12}$ The genetic material of microbes has followed us on our exodus from the birthplace of the human race in Africa and has been used alongside human markers to trace migration routes across the planet. ${ }^{11}$ In fact, detailed examination of strains of Helicobacter pylori may allow us to distinguish more accurately between human populations than a comparison of human genetic markers. ${ }^{11}$

Throughout human evolution, our environment has continuously shaped the composition of our microbiome, increasingly so during Neolithic, industrial revolution and modern eras. ${ }^{13}$ The use of fire, invention of agriculture, increased access to processed foods, including refined sugar after the industrial revolution, and the advent of antimicrobial therapy, are all likely to have influenced the composition of the human microbiome. ${ }^{13}$

\section{Temporal changes in the oral microbiome}

A study of calcified dental plaque samples from the time of transition from hunter-gather to Neolithic societies, and from the industrial revolution has proposed a compositional shift and declining microbial diversity around each of these evolutionary milestones..$^{14}$ It is, however, reasonable to point out that there are limitations of microbiome determinations from ancient preserved samples compared with viable microbes sampled in modern day, ${ }^{15}$ and these findings need to be interpreted with care. Introduction of refined sugar to our diet in the early times of agriculture caused certain oral bacteria to genetically evolve their metabolism to adapt to 'post-agricultural' changes in our diet. For example, Streptococcus mutans was able to successfully compete against other oral bacterial species by developing defences against increased oxidative stress and resistance against the acidic by-products of its own new efficient carbohydrate metabolism. ${ }^{16}$ This adaptation led to its increased prevalence in the oral cavity, along with other acid-tolerant species. In addition, since the industrial revolution, humans have been more frequently exposed to agents such as heavy metals, disinfectants, biocides and antibiotics that have the potential to eradicate or debilitate many microorganisms, while positively selecting for those microbes that carry resistance determinants. ${ }^{13}$ Oral hygiene practice changed towards the end of the nineteenth century in the developed world, mainly prompted by the publication of Willoughby Miller's book 'Microorganisms of the human mouth' in 1890, which generated a worldwide promotion of tooth brushing and flossing. ${ }^{17}$ This, too, is likely to have been a major factor in changes in the composition of the oral microbiome..$^{18}$ The modern-day excessive consumption of acidic drinks and refined sugar or cigarette smoking has further impacted on the oral ecosystem, ${ }^{14}$ leading to diseases such as caries and periodontal disease.

\section{The human microbiome is a complex ecological community}

We know now that the microorganisms (the microbiota) that make up the human microbiome are not just unicellular organisms living alongside each other, but instead form highly regulated, structurally and functionally organised communities attached to surfaces as biofilms, ${ }^{19}$ with interspecies collaborations as well as antagonisms that contribute to ecologic stability. Bacteria within a biofilm can communicate with each other by producing, detecting and responding to small diffusible signal molecules in a process called quorum sensing, which confers benefit for host colonisation, biofilm formation, defence against competitors and adaptation to changes in the environment. ${ }^{19}$ Quorum-sensing activities in biofilms are also involved in the virulence and pathogenic potential of bacteria and are therefore an important factor in understanding and controlling bacterial infections, ${ }^{19}$ as they enable microorganisms in biofilms to become more tolerant of host defences and antimicrobial agents. ${ }^{20}$

The endogenous human microbial communities contribute to critical metabolic, physiological and immunological functions, including: ${ }^{21-24}$

- Differentiation and maturation of the host mucosa and its immune system

- Food digestion and nutrition

- Energy generation

- Metabolic regulation and control of fat storage

- Processing and detoxification of environmental chemicals

- Maintenance of skin and mucosa barrier function

- Development and regulation of the immune system and fine-tuning of its reaction pattern, that is, the balance between proinflammatory and anti-inflammatory processes

- Prevention of invasion and growth of disease-promoting microorganisms (colonisation resistance).

Perturbations to the function and composition of the microbiome can have significant consequences for human health. ${ }^{7}$

The composition of our microbiome shows great diversity between compartments in the body, and is highly variable within and between people. ${ }^{25}$ It has been suggested that a person's gut bacteria and the community of microbes that live in the body and on the skin are distinct enough from the rest of the population to be used as a unique microbial 'fingerprint. ${ }^{26}$ Nonetheless, genetic variation in the human host is a dominant source of variation in composition of the microbiome, and twins share more similarities between their gut microbiomes than a twin and a parent or a non-twin sibling. ${ }^{27}$ Despite variations in the composition 
of the microbiomes between individuals, it is important to note that the overall functions of their microbiota are relatively consistent. ${ }^{13}$

Different body sites support entirely distinct microbial communities due to differences in prevailing biological and physical properties of each location/habitat. ${ }^{25}$ High specificity per body site was demonstrated by Kuczynski et al.: by using only ten randomly selected sequences from microbiome datasets, they could discriminate among the different body sites from which the samples were taken. ${ }^{28}$ The most diverse bacterial populations are found in the gastrointestinal tract and the mouth. ${ }^{29}$

\section{Special considerations for the oral microbiome}

The mouth is not a homogeneous environment for the resident microbiota, but offers several distinct habitats for microbial colonisation, ${ }^{30}$ such as teeth, gingival sulcus, attached gingiva, tongue, cheek, lip, and hard and soft palate. These oral habitats form a highly heterogeneous ecological system and support the growth of significantly different microbial communities. ${ }^{31}$ The warm and moist environment in the mouth suits the growth of many microorganisms and offers host-derived nutrients, such as saliva proteins, glycoproteins and gingival crevicular fluid (GCF). ${ }^{32}$ The teeth are the only natural non-shedding surfaces in the human body and provide unique opportunities for extensive biofilm formation, and a secure haven for microbial persistence. ${ }^{33}$ Dental restorations, crown and bridgework, removable prostheses and implants constitute additional non-shedding surfaces in the mouth that can influence biofilm formation and composition. ${ }^{34-36}$

To date, more than 700 prokaryotic taxa have been detected in the oral cavity, many of which cannot be isolated by common culture methods. ${ }^{37}$ Approximately $54 \%$ are validly named species,
$14 \%$ are unnamed (but cultivated) and 32\% are known only as uncultivated phylotypes. ${ }^{37}$ However, in any one individual, the number of resident species is estimated to be fewer. A detailed study of 9 oral sites in 26 subjects using DNA sequencing revealed a mean of 296 specieslevel taxa in each individual, while 557 taxa were found among the 26 subjects (unpublished data; Dr Floyd Dewhirst, personal communication).

\section{Acquisition of a normal oral microbiome}

During birth, the mother transmits microbes to the child, and delivery mode (vaginal versus caesarean) is therefore a determinant for the type of microorganisms that a child is initially exposed to. ${ }^{38}$ Delivery mode also influences the diversity of the oral microbiome later on in an infant's life, with vaginally-born children showing a higher number of taxa 3 months after birth compared with children born by caesarean section. ${ }^{39}$ The method of feeding also has an effect, with 3-month-old breast-fed infants showing a higher colonisation with oral lactobacilli than formulafed infants. ${ }^{40}$ The eruption of teeth provides new surfaces for microbial colonisation and constitutes a major ecological event in the mouth of a child. ${ }^{41}$ By the age of three, the oral microbiome of children is already complex, and becomes increasingly so with age. ${ }^{42}$ Replacement of the primary teeth with an adult dentition again significantly alters the oral microbial habitat. ${ }^{31}$

\section{Maintaining a healthy oral microbiome}

Once established, the oral microbiome is maintained by host- and microbe-derived factors, involving processes that are still not fully understood. Resident bacteria have both pro- and anti-inflammatory activities that are crucial for maintaining homeostasis at heavily colonised sites such as the oral cavity. ${ }^{43}$ Due to the interplay of the host's immune system with its microbial symbionts, acute infections of the oral mucosa are rather rare, despite dense microbial colonisation. ${ }^{44}$ The importance of these host-microbe interactions is highlighted by observations in immunosuppressed patients, who can experience life-threatening viral and fungal infections of the mucous membranes and oral infections by non-oral species. ${ }^{45-47}$

Both saliva and GCF provide nutrients for microbial growth and contain components with antimicrobial activities. ${ }^{32,48,49}$ The role of saliva in promoting oral health is well established. ${ }^{32}$ In addition to facilitating mastication, swallowing and speech, and aiding digestion, saliva contains vital enzymes and proteins that help maintain a balanced microbiota. Up to $10^{8}$ microorganisms have been detected per millilitre of saliva, mostly derived from oral mucosal surfaces such as the tongue. ${ }^{50}$ Salivary components are the primary nutritional source for microorganisms, and are required for the development of a balanced microbiome. A large number of salivary components, including secretory immunoglobulin A, lactoferrin, lactoperoxidase, lysozyme, statherin and histatins, directly and indirectly regulate the microbiome, keeping it in balance. ${ }^{32}$ For example, lactoperoxidase catalyses the production of hypothiocyanite from hydrogen peroxide - a product of bacterial metabolism - and saliva-secreted thiocyanate. Hypothiocyanite exerts direct antimicrobial effects by inhibiting bacterial glycolysis. ${ }^{51}$ Another salivary component with antimicrobial potential is nitrite, converted from dietary nitrates by oral bacteria. Nitrite is further reduced to nitric oxide that can inhibit growth of cariogenic bacteria and thus may help to protect against caries. ${ }^{52}$

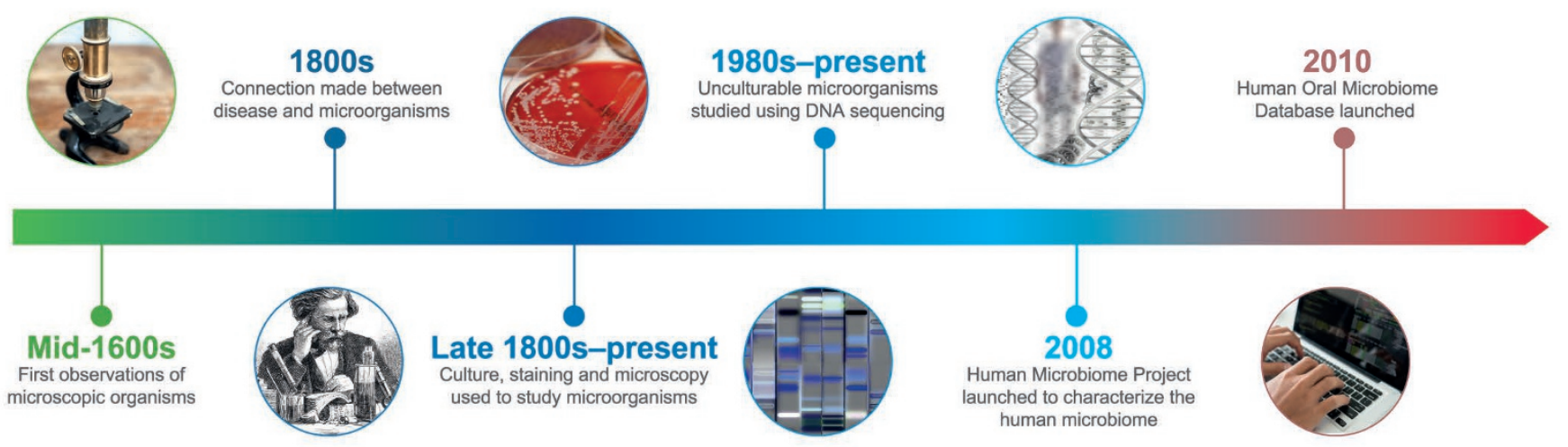

Fig. 3 Evolution of microbiological methods over time 
Proteins, including enzymes, lipids and other components (carbohydrates, nucleic acids), mainly from saliva, but also derived from GCF, the oral mucosa and bacteria, form the acquired pellicle, which modulates attachment of bacteria to dental and epithelial surfaces and protects the tooth surfaces against acid attacks. ${ }^{53}$ Enzymes that help to regulate the balance of the microbiome are immobilised in the acquired pellicle in an active conformation. ${ }^{54}$ The individually composed acquired pellicle triggers and mediates bacterial adherence to the non-shedding tooth surfaces via various interactions.

Saliva not only helps to maintain an environment that allows biofilms to flourish, but also modulates the layers of plaque with the help of numerous proteins, including enzymes and glycoproteins, and minerals, which control biofilm build-up and activity. ${ }^{32,55}$ Plaque biofilm is also dislodged by movement of the oral muscles of the cheeks and tongue during speech and mastication and by the flow of saliva.

\section{Characterisation of the oral microbiome}

A variety of conventional methods have been used to analyse the composition of the oral microbiome, including microscopy, cultural analysis, enzymatic assays and immunoassays. ${ }^{56-58}$ Figure 3 provides an overview of how microbiological methods have evolved over the years. However, many oral bacteria are fastidious and slow-growing, and require complex growth media, specific atmospheric requirements and long incubation times. Many oral bacteria are strict anaerobes, and expert care must be taken in sample collection, transport and incubation to prevent exposure to oxygen. Comprehensive cultural analysis of samples is difficult and only allows for the processing of small sample numbers. Selective bacteriological media have proven useful for studying particular species of interest, but may have biased our understanding of microbial aetiology of oral disease, attributing disease characteristics to species that happen to thrive under such culture conditions, while others remain undetected.

The advent of culture-independent methods has greatly improved the detection of microorganisms, many of which cannot yet be grown in culture. ${ }^{57}$ The most common culture-independent technique to analyse the microbiome is based on $\mathbf{1 6 S}$ ribosomal RNA (16S rRNA) gene community profiling. ${ }^{57}$ The $16 \mathrm{~S}$ rRNA gene is present in all prokaryotes and contains variable regions that are unique between microorganisms and that can be used as a means of identification. 16S rRNA genes can be extracted from heterogeneous samples, amplified and sequenced, and then compared with databases such as the Human Oral Microbiome Database, ${ }^{37}$ a publically available online resource that links sequence data with phenotypic, phylogenetic, clinical and bibliographic information of microorganisms found in the oral cavity. If a match for the sequence is found on the database, the microorganism can be identified; if there is no matching entry on the database, the sequence can be added as a record for a previously unknown phylotype.

The traditional method of 16S rRNA gene sequencing was costly, laborious and time-consuming. The advent of NGS methods such as 454 pyrosequencing (which is currently being phased out) and Illumina MiSeq have enabled a massively increased sample throughput, with up to 27 million sequences being generated in a single run (compared with a few hundred with the traditional method). A more detailed description of NGS is shown in Figure 4. The simplicity and relative affordability of NGS has led to enormous data generation and an explosion in publications, with accompanying challenges for data analysis and interpretation. Great care has to be taken in the conduct of NGS studies to avoid contamination of clinical samples with bacterial DNA present in some extraction kits, laboratory reagents and sample collection tools, which can significantly influence the outcome of studies. ${ }^{59,60}$ Despite these caveats, NGS is a useful tool that allows for high-volume studies of the genetic material in samples and has greatly increased our knowledge and understanding of the oral microbiome.
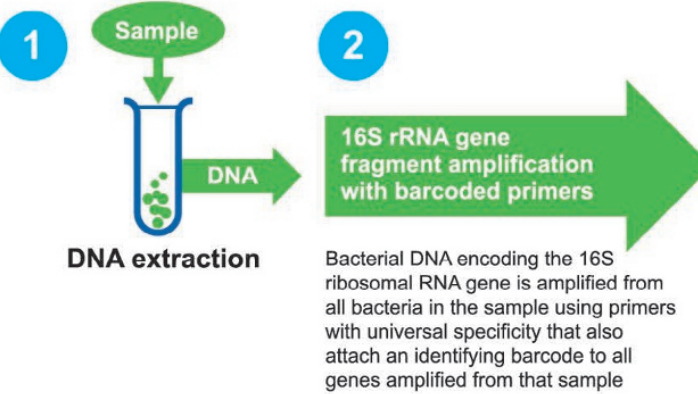

attach an identifying barcode to all

9

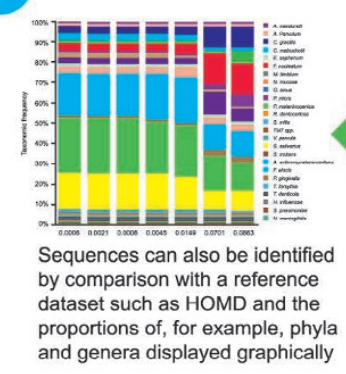

8

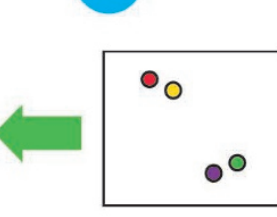

The composition of the samples can then be compared and displayed graphically
3

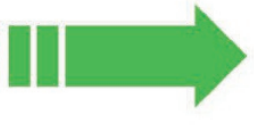

The amplified genes are purified and mixed together in equal proportions
7

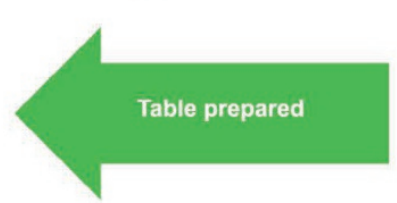

A table is prepared that shows the relative

abundance of each OTU

in each sample

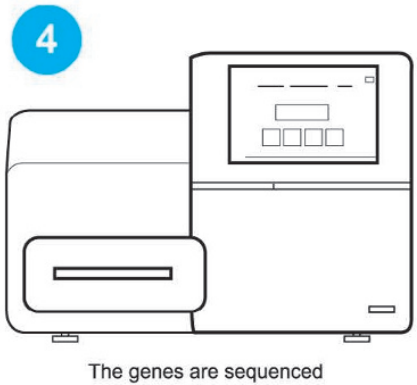

5

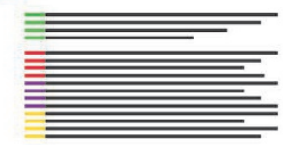

The sequences are separated by sample by reading the barcodes

Fig. 4 Overview of the next-generation sequencing pipeline 
Other modern approaches include metagenomics and metatranscriptomics. While metagenomics provides insights into the genetic composition of the microbial community in a sample, metatranscriptomics allows researchers to study the actively transcribed genes. ${ }^{61}$ Both methods are, to date, still hampered by technical challenges, but provide great opportunities to further our knowledge of the collective genome of the oral microbiome and its metabolic actions in the future.

Culture-independent methods have provided great insight into the diversity of the microbiome, but to investigate the properties and potential of an organism, it needs to be grown in culture. Currently, up to a third of the species in the oral microbiome are only known by their $16 \mathrm{~S}$ rRNA gene sequence, ${ }^{62}$ and there is an ongoing quest to develop new methods for growing currently 'uncultivatable' microorganisms. ${ }^{62}$ Progress in this area has been made, and a new method using siderophores (small, highaffinity iron-chelating compounds secreted by microorganisms such as bacteria, fungi and grasses) has been developed to isolate and grow novel strains. ${ }^{62}$ Some isolates depend on 'helper strains' for successful culture, implying their dependency on quorum-sensing and nutritional and/or signalling interactions with other bacteria within the biofilm community that they naturally inhabit. ${ }^{62}$

The application of such novel culturing methods, along with emerging molecular biological and bioinformatics approaches and increased computational power, will not only grow our understanding of the oral microbiome, but also help us devise interventional strategies to maintain health and target disease in future.

\section{An unbalanced oral microbiome may be detrimental to general health}

The oral microbiota contributes to oral and general well-being (Fig. 1), and its loss can be detrimental to the health of the individual. An example of this is the effect of nitrate-reductase-expressing oral bacteria, which have been shown to catalyse the conversion of dietary nitrates to nitrite. After being swallowed, salivary nitrite is further converted to nitric oxide, a potent vasodilator with antimicrobial activity which plays a critical role in sustaining cardiovascular health. ${ }^{63}$ Nitrite also stimulates gastric mucus production. ${ }^{64} \mathrm{~A}$ modest consumption of nitrate has been found to lead to a reduction of blood pressure, inhibition of platelet function and reduced endothelial
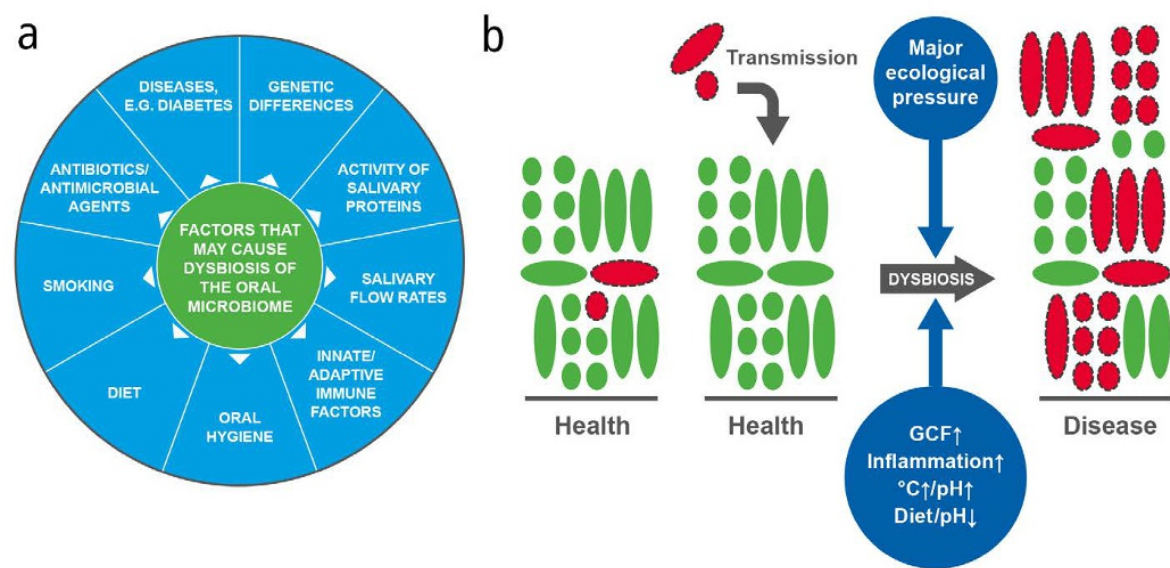

Altered competitiveness

\section{Fig. 5 (a) Causes of dysbiosis; (b) A model of dysbiosis (adapted from Marsh ${ }^{80}$ )}

In health, the majority of the bacteria have a symbiotic relationship with the host; for simplicity, these microorganisms are shown in green. Potentially cariogenic or periodontopathic bacteria (shown in red with dotted outlines) have been detected at healthy sites at low levels that are not clinically relevant; they may also be acquired from close partners (transmission), but again, their levels would be extremely low relative to the bacteria associated with health. In disease, there is an increase in the numbers and proportions of cariogenic or periodontopathic bacteria, and there may be increased biomass (especially in gingivitis). It is proposed that for this to happen, there has to be a change in local environmental conditions (major ecological pressure), which alters the competitiveness of bacteria within the biofilm and selects for those species that are most adapted to the new environment. The factors driving this selection need to be recognised and addressed for adequate and consistent disease prevention. dysfunction. ${ }^{65}$ A recent study has found that sustained ingestion of dietary nitrates improves vascular function in patients with hypercholesterolaemia. ${ }^{66}$ These improvements were associated with changes in the oral microbiome in favour of organisms that are capable of nitrite reduction. ${ }^{66}$ However, while dietary nitrates are able to boost nitrite formation, it is also the case that nitric oxide in tissues can interact with superoxide radicals released by immune cells to form peroxynitrite anions, which have been suggested to have detrimental actions on cells, such as DNA damage. ${ }^{67}$ The subject of the effects of nitrate/nitrite/nitric oxide on health still evokes some controversy; however, several small-scale studies have shown that the use of chlorhexidine-containing mouthwashes can reduce the concentration of nitrites in both saliva and plasma and lead to a small rise in blood pressure. ${ }^{68-70}$ While these findings are interesting, it is important to note that confirmation from larger-scale studies is necessary before any firm conclusions can be drawn.

\section{Dysbiosis: the oral microbiome in disease}

The complex equilibrium between resident species in the oral cavity is responsible for the maintenance of a healthy state (in symbiosis) or a state associated with disease (in dysbiosis). A dysbiotic microbiome is one in which the diversity and relative proportions of species or taxa within the microbiota is disturbed. ${ }^{7}$ The relationship between the oral microbiome and its host is dynamic and, while in the healthy mouth the composition of microbial communities is remarkably stable (after the microbiome has matured in childhood), biological changes in a person's life can affect the balance of the species within these communities. ${ }^{71}$ These include physiological changes, for example, age, or hormonal changes in puberty and pregnancy, to which healthy individuals can often adapt without detriment to their oral health. ${ }^{72}$ At other times, the finely-tuned ecosystem in the mouth can become disturbed, causing a dysbiotic shift and a loss of community balance or diversity in the biofilm, ${ }^{73}$ with a single or few species predominating, and an associated increased risk of disease. Modifiable factors driving oral dysbiosis include salivary gland dysfunction (that is, changes in saliva flow and/or composition), poor oral hygiene, gingival inflammation and lifestyle choices, including dietary habits and smoking. ${ }^{74,75}$ Figure 5 a gives a summary of factors contributing to dysbiosis. 
It is now an accepted concept that the bacteria historically considered as oral 'pathogens' can be found in low numbers at healthy sites, and oral disease occurs as a consequence of a deleterious change to the natural balance of the microbiota rather than as a result of exogenous 'infection. ${ }^{\text {?1 }}$ In dysbiosis, these disease-associated bacteria can grow to markedly higher proportions than under healthy conditions, where they are normally minor and innocuous components in the biofilm. ${ }^{71}$

Alterations in the pattern of biofilm formation may result in dysbiotic microenvironments in the many distinct habitats in the mouth. The distinct, non-shedding structure of teeth (smooth surfaces, pits and fissures, proximal sites and exposed root surfaces) enables large masses of microbes to accumulate as dental plaque biofilm. ${ }^{76}$ Therefore, the plaque biofilm is not naturally shed as it accumulates, which is likely a key driver of dysbiosis in the absence of oral hygiene to disrupt and remove it.

\section{Dysbiosis as origin of caries and periodontitis}

Different theories on the relationship between plaque and dental disease have evolved over time. ${ }^{77}$ The 'nonspecific plaque hypothesis' (NSPH) has its origins in the nineteenth century, but was further refined over the next hundred years. Initially, the NSPH speculated that dental infections were caused by the nonspecific overgrowth of all bacteria in dental plaque. The NSPH was further extended to stipulate that destructive periodontitis was the result of subgingival colonisation, favoured by ecological changes associated with plaque accumulation, gingivitis and gingival exudate. These changes increase the numbers of microorganisms and alter their proportions, but no single species appears in active sites that is not also commonly present in inactive sites. ${ }^{78}$ Since it was assumed that any plaque had the potential to cause disease, it was concluded that nonspecific mechanic removal of as much plaque as possible, for instance by tooth brushing or interdental cleaning, would be the best way of disease prevention. ${ }^{77}$

Evolving laboratory techniques allowed for the isolation and characterisation of more species in culture. The observation that kanamycin was particularly effective against caries-associated species, such as streptococci, led to the emergence of the 'specific plaque hypothesis', which proposed that only a few species in the oral microbiome are involved in the disease process, and that targeting these species with antibiotics could cure or prevent disease (initially caries and later periodontitis). ${ }^{77,79}$ However, results from clinical studies employing antibiotics for the treatment of both conditions have been largely disappointing in terms of translation to day-to-day practice and magnitude of long-term clinical benefit. ${ }^{77}$ These outcomes could possibly be explained by the fact that, as mentioned before, a large proportion of microorganisms cannot be grown in culture, and thus bias may have been introduced by the isolation of only those that are cultivatable.

Following a renewed interest in the NSPH in the 1980s, an ecological plaque hypothesis was proposed to explain the relationship between the resident oral microbiota, the host environment and oral diseases (Fig. 5b). ${ }^{80}$ Substantial changes in a local environment can alter the competitiveness of plaque bacteria, leading to the enrichment of organisms most suited to the new environment. ${ }^{80}$ This hypothesis introduces the concept that disease can be prevented not only by directly inhibiting the putative pathogens, but also by interfering with the environmental factors driving the selection and enrichment of these bacteria. ${ }^{80}$ For instance, in caries, an increased frequency of sugar intake, or a reduction in saliva flow, results in plaque biofilms that are exposed for longer and more regular periods to lower $\mathrm{pH}$ levels. This selects for organisms that produce acids themselves and/or are more tolerant of an acidic environment, at the expense of bacteria that thrive in neutral conditions or contribute to $\mathrm{pH}$ neutralisation. ${ }^{71,81}$ A contemporary model of host-microbe interactions in the pathogenesis of caries is shown in Figure 6.

The ecological plaque hypothesis was further refined by the proposal that certain low-abundance microbial pathogens can cause inflammatory disease by interfering with the host immune system and remodelling of the microbiota, leading to gingivitis and

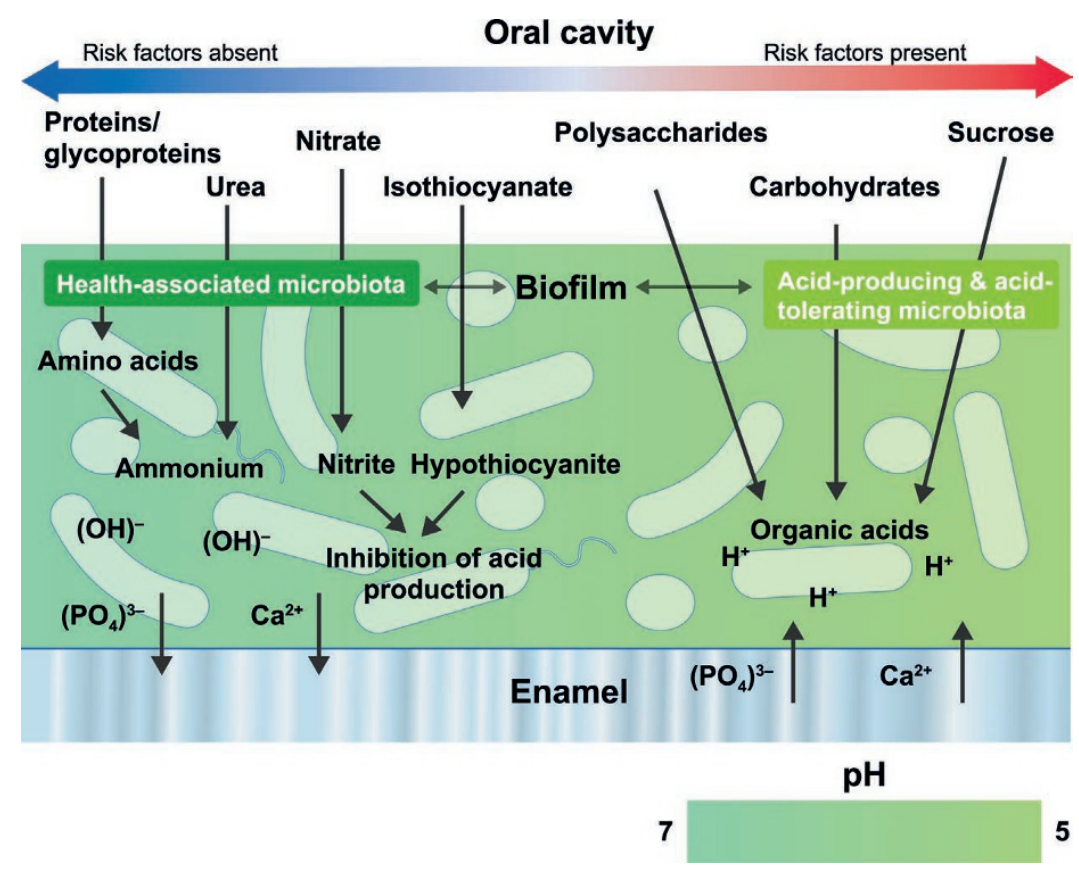

Fig. 6 A contemporary model of host-microbe interactions in the pathogenesis of caries (adapted from de Soet \& Zaura and Takahashi) ${ }^{97,51}$

In caries, carbohydrates are fermented to organic acids (for example, lactic acid), which lower the local pH resulting in net demineralisation of the tooth surface. This drives the selection of efficient acid-producing and acid-tolerating bacteria (dysbiosis). In contrast, in health, there is a more complex and balanced pattern of metabolism involving the catabolism of salivary proteins and glycoproteins, with the generation of alkali to neutralise any acid produced. The biofilm undergoes multiple pH cycles during the day, resulting in enamel de- and remineralisation (showed by ion efflux and influx into the enamel in the diagram). If fluoride ions are present in the biofilm, $\mathrm{F}$ - is then taken up in the superficial layer of enamel during the remineralisation phase, slowing down demineralisation during the acid challenge. Only when there are too many risk factors (for example, not sufficient time for $\mathrm{pH}$ neutralisation due to too frequent snacking, plaque being undersaturated with regard to fluoride, calcium and phosphate, low salivary clearance), demineralisation outweighs remineralisation and results in a net mineral loss with, in due time, enamel lesions. 
Behavioural risk factors absent

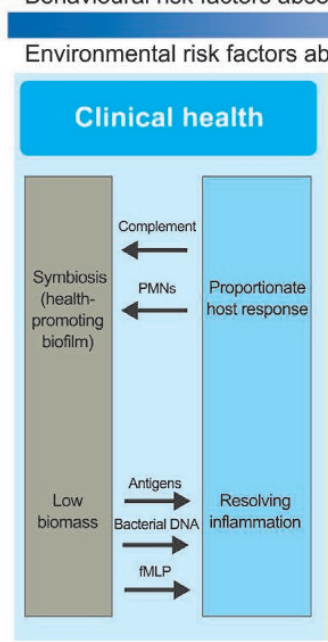

Genetic risk factors absent

Epigenetic effects not evident
Behavioural risk factors present

Environmental risk factors evident
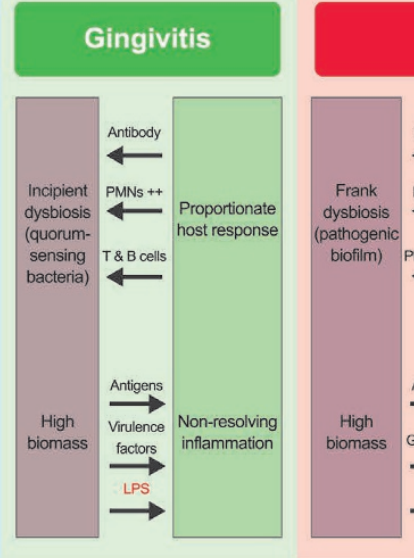

Genetic risk factors present
Periodontitis

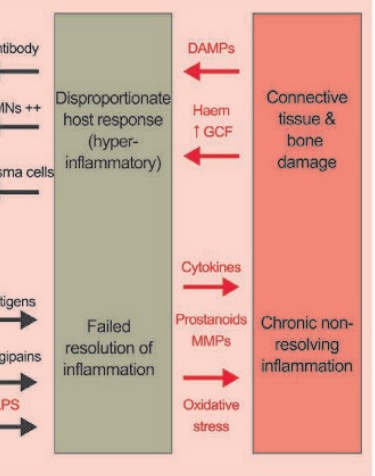

Epigenetic effects evident

Chapple 2015

Fig. 7 A model of periodontitis pathogenesis (adapted from Meyle \& Chapple) ${ }^{82}$ that has evolved from the classical model of Page \& Kornman ${ }^{98}$

Clinical health assumes some mild inflammatory changes that are proportionate to maintaining a resident 'health-promoting' periodontal microbiota. The relationship is symbiotic, with the host living harmoniously with its microbes. However, if the biofilm is allowed to accumulate and is not regularly disturbed/removed, certain pathogenic bacteria are able to emerge and an 'incipient dysbiosis' develops. For example, Porphyromonas gingivalis requires iron from haem and can start to establish itself and contribute to the dysbiosis, because as local inflammation increases it provides iron from gingival bleeding. In gingivitis, the host response remains proportionate, but due to the maturing biofilm, the associated inflammation does not resolve so easily and becomes chronic in nature, supporting the dysbiosis. Dependent upon various genetic, environmental and lifestyle risk factors, some susceptible patients will progress to periodontitis. The latter is driven by disproportionate and exaggerated host inflammatory immune responses that cause the majority of host tissue damage and drive a frank dysbiosis with failed resolution of the chronic destructive inflammation. A vicious cycle develops that sustains the disequilibrium, but symbiosis may be restored by regular and thorough biofilm disruption to help resolve the inflammation. DAMP - damage-associated molecular pattern; fMLP - f-Met-Leu-Phe; GCF - gingival crevicular fluid; LPS - lipopolysaccharide; MMP - matrix metalloproteinase; PMN - neutrophilic polymorphonuclear leucocyte.

periodontitis. Figure 7 shows a contemporary model of host-microbe interactions in the pathogenesis of gingivitis and periodontitis. ${ }^{82}$ Accumulation of biofilm triggers gingivitis; however, the presence of biofilm alone is not sufficient to advance into periodontitis. It is now recognised that complex interactions between immune response mediators and the biofilm are necessary requirements that lead to disease progression from gingivitis to periodontitis. ${ }^{82}$ A dysbiotic microbial community subverts the host response so that most tissue damage is due to an inappropriate and uncontrolled level of inflammation. ${ }^{82-84}$ Local inflammation resulting from biofilm accumulation causes an increased flow of the nutrient-rich GCF and potentially bleeding, whereby the site becomes deprived of oxygen, favouring the growth of anaerobic microbes. ${ }^{71}$ The inflammatory changes in the periodontal environment provide an ideal environment for the growth of obligatory anaerobic and proteindependent bacteria that reside in the gingival crevice, driving a shift from a symbiotic microbiome to dysbiosis. ${ }^{71}$ Inflammation-induced micro-ulceration of the sulcular epithelium results in the leakage of blood (and thereby iron) into the gingival crevice. The resulting conditions are conducive for periodontitisassociated species such as Porphyromonas gingivalis and Aggregatibacter actinomycetemcomitans to survive and flourish. ${ }^{85,86}$ The dysbiotic microbiota induces the destruction of the periodontal tissue by a dysregulated host inflammatory immune response, which in turn provides new tissue breakdown-derived nutrients for the bacteria. ${ }^{87}$ Through evolution, bacteria have adapted to take advantage of inflammation-derived nutrients, promoting further dysbiosis and tissue disruption, creating a self-perpetuating pathogenic cycle. ${ }^{87}$

The degree by which biofilm accumulation promotes periodontitis varies between individuals, ${ }^{82}$ according to their risk profile. In patients not susceptible to periodontitis, the inflammatory response in gingivitis is proportionate and selfresolving, but in susceptible patients, multiple genetic, epigenetic or patient-modifiable factors (tobacco, alcohol, diet, unregulated diabetes, stress, etc) can trigger an exaggerated, yet ineffective and chronic, non-resolving inflammation in the connective tissues supporting the teeth. ${ }^{82}$ This is even more aggravated in diabetes, which is associated with metabolic dysregulation that modulates the inflammatory response, leading to a highly inflammatory state that further accelerates tissue destruction and attachment loss. ${ }^{82}$

Although, to date, it is well established that particular microbial communities shift in response to disease, it remains to be elucidated if there is a 'healthy amount' of these bacteria that is consistent across individuals, ${ }^{88}$ or whether this differs between individuals due to different thresholds for activation and/ or deregulation of the host response.

\section{Systemic consequences of oral dysbiosis}

The coevolution to a harmonious coexistence is only valid as long microbes remain in their natural habitat and are not disseminated to other body sites, where they can cause disease. Dysbiosis in periodontal disease as a trigger of bacteraemia likely facilitates systemic dissemination of oral bacteria, and good oral hygiene is therefore crucial for controlling the total bacterial load. ${ }^{89,90}$ The link between oral pathogens and systemic effects has been evidenced by a recent study in animals, which found a direct effect of oral administration of $P$. gingivalis on the composition of the gut microbiome as well as inflammatory changes in various tissues and organs. ${ }^{91}$ Oral bacteria have been proposed to play a role in a number of systemic diseases, including cardiovascular disease, rheumatoid arthritis, adverse pregnancy outcomes, stroke, inflammatory bowel disease and colorectal cancer, respiratory tract infection, meningitis or brain abscesses, lung, liver or splenic abscesses, appendicitis, pneumonia and diabetes. . $^{30,58,90,92,93}$

It is well established that severe periodontitis adversely affects glycaemic control in diabetes and glycaemia in subjects who do not have diabetes. Severe periodontitis poses an increased 
risk for the onset of type 2 diabetes, and there is a direct and dose-dependent relationship between the severity of periodontitis and diabetic complications. ${ }^{92}$ However, more research is needed to determine whether the periodontal microbiota has any direct impact on diabetes.

Analysis of the oral cavity and its microbiome may be a useful tool to diagnose systemic diseases that have periodontal manifestations. Good oral hygiene to control the total microbial load is important to prevent dissemination to other body sites. ${ }^{90}$

\section{Conclusions}

Improved understanding of the holobiont - current and future implications for clinical practice

The diverse community that makes up the oral microbiome is finely tuned by nature to protect from disease, and it is of great importance to maintain its natural diversity. Modern lifestyles can disturb and upset the natural balance of our oral microbiome, and our clinical goal should be to re-establish its symbiotic equilibrium by whatever means are necessary and appropriate in the individual patient. Thus, it is pivotal that both patients and healthcare professionals embrace the concept of a balanced oral microbiome and its importance in oral and systemic health. Treatment sessions should include prevention strategies, such as advice on oral hygiene practices and on diet and smoking. As a first approach, active maintenance of health rather than management of disease should be the primary concern for caregivers and patients alike. Oral healthcare professionals can achieve this by educating patients on appropriate lifestyle choices and the application of effective plaque control techniques that preserve dental biofilms at levels compatible with oral health. This approach allows for the beneficial properties of the resident microbiota to be maintained while the risk of dental disease from excessive plaque accumulation is reduced. Once dysbiosis occurs, the treatment goal should be to re-establish the lost harmonious balance by keeping good oral hygiene and modifying lifestyle factors such as diet and smoking. Indiscriminate use of antibiotics for the treatment of oral diseases should be avoided, aiming to safeguard the beneficial oral microbiota and avoid antibiotic resistance. For the control of caries, in addition to use of topical fluoride, measures should be taken that encourage a shift away from an acidic environment, through reduction in the amount and frequency of the consumption of sucrose and acidic drinks (even if the latter are sugarfree), together with supplementation with agents that can reduce acid production and/ or promote alkali generation within dental plaque. For periodontal disease, treatment strategies should aim for mechanical reduction of accumulated biofilm by mechanical removal of plaque back to levels compatible with oral health. This would reduce inflammation and the accompanying flow of GCF, and promote a favourable microenvironment to support formation of a balanced microbiome. Where relevant, patients should also be advised on smoking cessation and control of diabetes.

A small study has recently shown that the composition of the subgingival microbiome at baseline, rather than the use of antibiotics, has a predictive potential on the long-term clinical outcome for treatment of chronic periodontitis. ${ }^{94}$ While further research is needed to confirm details of predictive value, the finding underlines the important role of the oral microbiome, not only in disease, but also in the response of oral tissues.

Our current understanding of this rapidly evolving scientific field supports the notion that clinical practice needs to shift from its historical focus on management of caries and periodontitis by elimination of the microbiota, to a new focus on proactive management of oral health through an ecological approach to the holobiont. Future directions may include individual assessment of the microbiome and the host response for the early detection of subjects at high risk, and personalised approaches to restore a health-associated oral microbiome after dysbiosis - potentially tantalising developments that would have direct implications for patient management in clinical practice.

Support for third-party writing assistance for this manuscript, furnished by Dr Kristina Standeven of MediTech Media UK, was provided by Unilever.

\section{Duality of interest}

MK has given scientific presentations funded by Unilever. ILCC has received research funding from a number of oral care companies, including Unilever. $M H$ has advised, and received research funding from, a number of oral care and dental companies. PDM has advised, and spoken at conferences funded by a number of oral care companies. VM has received research funding from a number of oral care companies. AMLP has advised, and received research funding from a number of oral care companies. MST has received research funds and consulting fees from a variety of oral healthcare companies. WGW has advised, and received research funding from, a number of oral care companies. EZ has received research funding from a number of oral care companies.
1. Bordenstein $S R$, Theis K R. Host biology in light of the microbiome: ten principles of holobionts and hologenomes. PLoS Biol 2015; 13: e1002226.

2. Sender R, Fuchs $S$, Milo R. Are we really vastly outnumbered? Revisiting the ratio of bacterial to host cells in humans. Cell 2016; 164: 337-340.

3. Lederberg J, Mccray A. 'Ome sweet 'omics - a genealog ical treasury of words. Scientist 2001; 15: 8-10.

4. Turnbaugh P J, Ley R E, Hamady M et al. The human microbiome project. Nature 2007; 449: 804-810.

5. MetaHIT Consortium (Metagenomics of the Human Intestinal Tract consortium). 2016. Available online at: http://www.sanger.ac.uk/resources/downloads/bacteria/ metahit/ (accessed November 2016)

6. The White House Office of Science and Technology Policy. Fact sheet: Announcing the National Microbiome Initiative. 2016. Available online at: https://www. whitehouse.gov/sites/whitehouse.gov/files/documents/ OSTP\%20National\%20Microbiome $\% 2$ Initiative $\% 20$ Fact\%20Sheet.pdf (accessed November 2016).

7. Cho I, Blaser M J. The human microbiome: at the interface of health and disease. Nat Rev Genet 2012; 13: 260-270.

8. Blaser M J. Who are we? Indigenous microbes and the ecology of human diseases. EMBO Rep 2006; 7: 956-960

9. Haubek D, Ennibi O K, Poulsen K et al. Risk of aggressive periodontitis in adolescent carriers of the JP2 clone of Aggregatibacter (Actinobacillus) actinomycetemcomitans in Morocco: a prospective longitudinal cohort study. Lancet 2008; 371: 237-242.

10. Ravel J, Gajer P, Abdo Z et al. Vaginal microbiome of reproductive-age women. Proc Natl Acad Sci U S A 2011; 108 (Suppl 1): 4680-4687.

11. Rinaldi A. Tiny travel companions. As microorganisms have accompanied mankind's journeys around the globe they could help scientists to unravel our past. EMBO Rep 2007; 8: 121-125.

12. Rylev M, Kilian M. Prevalence and distribution of principal periodontal pathogens worldwide. J Clin Periodontol 2008; 35: 346-361.

13. Gillings M R, Paulsen I T, Tetu S G. Ecology and evolution of the human microbiota: Fire, farming and antibiotics. Genes (Basel) 2015; 6: 841-857.

14. Adler C J, Dobney K, Weyrich L S et al. Sequencing ancient calcified dental plaque shows changes in oral microbiota with dietary shifts of the Neolithic and Industrial revolutions. Nat Genet 2013; 45: 450-455.

15. Costalonga M, Herzberg M C. The oral microbiome and the immunobiology of periodontal disease and caries. Immunol Lett 2014; 162: 22-38.

16. Cornejo O E, Lefebure T, Bitar P D et al. Evolutionary and population genomics of the cavity causing bacteria Streptococcus mutans. Mol Biol Evol 2013; 30: 881-893.

17. American Dental Association. History of Dentistry Timeline. 2016. Available online at: http://www.ada.org/ en/abouttheada/adahistoryandpresidentsoftheada/adahistoryofdentistrytimeline (accessed November 2016).

18. Marsh PD. Controlling the oral biofilm with antimicrobials. J Dent 2010; 38 (Suppl 1): S11-S15.

19. Li Y H, Tian X. Quorum sensing and bacterial social interactions in biofilms. Sensors (Basel) 2012; 12: 2519-2538.

20. Donlan R M, Costerton J W. Biofilms: survival mechanisms of clinically relevant microorganisms. Clin Microbiol Rev 2002; 15: 167-193.

21. Donohoe D R, Garge N, Zhang X et al. The microbiome and butyrate regulate energy metabolism and autophagy in the mammalian colon. Cell Metab 2011; 13: 517-526.

22. Krajmalnik-Brown R, Ilhan Z E, Kang D W, DiBaise J K. Effects of gut microbes on nutrient absorption and energy regulation. Nutr Clin Pract 2012; 27: 201-214.

23. Relman D A. The human microbiome: ecosystem resilience and health. Nutr Rev 2012; 70 (Suppl 1): S2-S9.

24. Relman D A. The human microbiome and the future practice of medicine. JAMA 2015; 314: 1127-1128

25. Costello E K, Lauber C L, Hamady M et al. Bacterial community variation in human body habitats across space and time. Science 2009; 326: 1694-1697.

26. Franzosa E A, Huang K, Meadow J F et al. Identifying personal microbiomes using metagenomic codes. Proc Natl Acad Sci U S A 2015; 112: E2930E2938.

27. Turnbaugh P J, Hamady M, Yatsunenko T et al. A core gut microbiome in obese and lean twins. Nature 2009; 457: 480-484. 
28. Kuczynski J, Costello E K, Nemergut D R et al. Direct sequencing of the human microbiome readily reveals community differences. Genome Biol 2010; 11: 210.

29. Human Microbiome Project Consortium. A framework for human microbiome research. Nature 2012; 486: 215-221.

30. Dewhirst F E, Chen T, Izard J et al. The human oral microbiome. J Bacteriol 2010; 192: 5002-5017.

31. Xu X, He J, Xue J et al. Oral cavity contains distinct niches with dynamic microbial communities. Environ Microbiol 2015; 17: 699-710.

32. van't Hof W, Veerman E C, Nieuw Amerongen A V, Ligtenberg A J. Antimicrobial defense systems in saliva. Monogr Oral Sci 2014; 24: 40-51.

33. Marsh P D, Devine D A. How is the development of dental biofilms influenced by the host? I Clin Periodontol 2011; 38 (Suppl 11): 28-35.

34. Busscher H J, Rinastiti M, Siswomihardjo W, van der Mei H C. Biofilm formation on dental restorative and implant materials. J Dent Res 2010; 89: 657-665.

35. Hannig C, Hannig M. The oral cavity - a key system to understand substratum-dependent bioadhesion on solid surfaces in man. Clin Oral Investig 2009; 13: 123-139.

36. Øilo M \& Bakken V. Biofilm and dental biomaterials. Materials 2015; 8: 2887-2900.

37. Human Oral Microbiome Database (HOMD). 2016 Available online at: http://www.homd.org/ (accessed April 2016).

38. Dominguez-Bello M G, Costello E K, Contreras M et al. Delivery mode shapes the acquisition and structure of the initial microbiota across multiple body habitats in newborns. Proc Natl Acad Sci U S A 2010; 107: 11971-11975.

39. Lif H P, Harnevik L, Hernell 0 et al. Mode of birth delivery affects oral microbiota in infants. J Dent Res 2011; 90: 1183-1188.

40. Holgerson P L, Vestman N R, Claesson R et al. Oral microbial profile discriminates breast-fed from formula-fed infants. J Pediatr Gastroenterol Nutr 2013; 56: 127-136.

41. Sampaio-Maia B, Monteiro-Silva F. Acquisition and maturation of oral microbiome throughout childhood: An update. Dent Res J (Isfahan) 2014; 11: 291-301.

42. Crielaard W, Zaura E, Schuller A A et al. Exploring the oral microbiota of children at various developmental stages of their dentition in the relation to their oral health. BMC Med Genomics 2011: 4: 22

43. Devine D A, Marsh P D, Meade J. Modulation of host responses by oral commensal bacteria. J Oral Microbio 2015; 7: 26941.

44. Zaura E, Nicu E A, Krom B P, Keijser B J. Acquiring and maintaining a normal oral microbiome: current perspective. Front Cell Infect Microbiol 2014; 4: 85

45. Petti S, Polimeni A, Berloco P B, Scully C. Orofacial diseases in solid organ and hematopoietic stem cell transplant recipients. Oral Dis 2013; 19: 18-36.

46. Soga Y, Maeda Y, Ishimaru F et al. Bacterial substitution of coagulase-negative staphylococci for streptococci on the oral mucosa after hematopoietic cell transplantation. Support Care Cancer 2011; 19: 995-1000.

47. Diaz P I, Hong B Y, Frias-Lopez J et al. Transplantation-associated long-term immunosuppression promotes oral colonization by potentially opportunistic pathogens without impacting other members of the salivary bacteriome. Clin Vaccine Immunol 2013; 20: 920-930.

48. Grant M M, Creese A J, Barr G et al. Proteomic analysis of a noninvasive human model of acute inflammation and its resolution: the twenty-one day gingivitis model. $J$ Proteome Res 2010; 9: 4732-4744

49. Barnes V M, Teles R, Trivedi H M et al. Acceleration of purine degradation by periodontal diseases. J Dent Res 2009; 88: 851-855.

50. Marsh P D, Do T, Beighton D, Devine D A. Influence of saliva on the oral microbiota. Periodontol 20002016 70: 80-92.
51. Takahashi N. Oral Microbiome Metabolism: From "Who are they?" to "What are they doing?". J Dent Res 2015; 94: 1628-1637.

52. Doel J J, Hector M P, Amirtham C V et al. Protective effect of salivary nitrate and microbial nitrate reductase activity against caries. Eur J Oral Sci 2004; 112: 424-428.

53. Siqueira W L, Custodio W, McDonald E E. New insights into the composition and functions of the acquired enamel pellicle. J Dent Res 2012; 91: 1110-1118.

54. Hannig C, Hannig M, Attin T. Enzymes in the acquired enamel pellicle. Eur J Oral Sci 2005; 113: 2-13.

55. Amerongen $A V$, Veerman $E C$. Saliva - the defender of the oral cavity. Oral Dis 2002; 8: 12-22.

56. Krishnan K, Chen T, Paster B J. A practical guide to the oral microbiome and its relation to health and disease. Oral Dis 2016.

57. Zarco M F, Vess T J, Ginsburg G S. The oral microbiome in health and disease and the potential impact on personalized dental medicine. Oral Dis 2012; 18: 109-120.

58. Pedersen A M (ed). Oral infections and general health: from molecule to chairside. Vol.1. Springer International Publishing, 2016.

59. Salter S J, Cox M J, Turek E M et al. Reagent and laboratory contamination can critically impact sequence-based microbiome analyses. BMC Biol 2014; 12 : 87.

60. van der Horst J, Buiijs M J, Laine M L et al. Sterile paper points as a bacterial DNA-contamination source in microbiome profiles of clinical samples. J Dent 2013; 41: 1297-1301.

61. Gilbert J A, Hughes M. Gene expression profiling: metatranscriptomics. Methods Mol Biol 2011; 733: 195-205.

62. Vartoukian S R, Adamowska A, Lawlor M et al. In vitro cultivation of 'unculturable' oral bacteria, facilitated by community culture and media supplementation with siderophores. PLoS One 2016; 11: e0146926.

63. Kapil V, Webb A J, Ahluwalia A. Inorganic nitrate and the cardiovascular system. Heart 2010; 96: 1703-1709.

64. Lundberg J O, Gladwin M T, Ahluwalia A et al. Nitrate and nitrite in biology, nutrition and therapeutics. Nat Chem Biol 2009; 5: 865-869.

65. Lundberg J O, Carlstrom M, Larsen F J, Weitzberg E. Roles of dietary inorganic nitrate in cardiovascular health and disease. Cardiovasc Res 2011; 89: 525-532.

66. Velmurugan S, Gan J M, Rathod K S et al. Dietary nitrate improves vascular function in patients with hypercholesterolemia: a randomized, double-blind, placebo-controlled study. Am J Clin Nutr 2016: 103: 25-38.

67. Chapple I L, Matthews J B. The role of reactive oxygen and antioxidant species in periodontal tissue destruction. Periodontol 2000 2007; 43: 160-232.

68. Woessner M, Smoliga J M, Tarzia B et al. A stepwise reduction in plasma and salivary nitrite with increasing strengths of mouthwash following a dietary nitrate load. Nitric oxide 2016; 54: 1-7.

69. Kapil V, Haydar S M, Pearl V et al. Physiological role for nitrate-reducing oral bacteria in blood pressure control. Free Radic Biol Med 2013; 55: 93-100.

70. Govoni M, Jansson E A, Weitzberg E, Lundberg J O. The increase in plasma nitrite after a dietary nitrate load is markedly attenuated by an antibacterial mouthwash. Nitric Oxide 2008; 19: 333-337.

71. Marsh P D, Head D A, Devine D A. Ecological approaches to oral biofilms: control without killing. Caries Res 2015; 49 (Suppl 1): 46-54

72. Zaura $E$, ten Cate J M. Towards understanding oral health. Caries Res 2015; 49 (Suppl 1): 55-61.

73. Gross E L, Leys E J, Gasparovich S R et al. Bacterial $16 \mathrm{~S}$ sequence analysis of severe caries in young permanent teeth. J Clin Microbiol 2010; 48: 4121-4128.

74. Marsh P D, Head D A, Devine D A. Prospects of oral dis ease control in the future - an opinion. J Oral Microbiol 2014; 6: 26176

75. Wu J, Peters B A, Dominianni C et al. Cigarette smoking and the oral microbiome in a large study of American adults. ISME J 2016; 10: 2435-2446.
76. Marcotte H, Lavoie M C. Oral microbial ecology and the role of salivary immunoglobulin A. Microbiol Mol Biol Rev 1998; 62: 71-109.

77. Rosier B T, De Jager M, Zaura E, Krom B P. Historical and contemporary hypotheses on the development of oral diseases: are we there yet? Front Cell Infect Microbiol 2014; 4: 92.

78. Theilade $E$. The non-specific theory in microbial etiology of inflammatory periodontal diseases. J Clin Periodontol 1986; 13: 905-911.

79. Loesche W J. Clinical and microbiological aspects of chemotherapeutic agents used according to the specific plaque hypothesis. J Dent Res 1979: 58: 2404-2412.

80. Marsh P D. Are dental diseases examples of ecological catastrophes? Microbiology 2003; 149: 279-294.

81. Liu Y L, Nascimento M, Burne R A. Progress toward understanding the contribution of alkali generation in dental biofilms to inhibition of dental caries. Int J Oral Sci 2012; 4: 135-140.

82. Meyle J, Chapple I. Molecular aspects of the pathogenesis of periodontitis. Periodontol 2000 2015; 69: 7-17.

83. Darveau R P. Periodontitis: a polymicrobial disruption of host homeostasis. Nat Rev Microbiol 2010; 8: 481-490.

84. Williams R C, Jeffcoat M K, Kaplan M L et al. Flurbiprofen: a potent inhibitor of alveolar bone resorption in beagles. Science 1985; 227: 640-642.

85. Bramanti T E, Holt S C. Roles of porphyrins and host iron transport proteins in regulation of growth of Porphyromonas gingivalis W50. J Bacteriol 1991; 173: 7330-7339.

86. Hajishengallis $\mathrm{G}$. Immunomicrobial pathogenesis of periodontitis: keystones, pathobionts, and host response. Trends Immunol 2014: 35: 3-11.

87. Hajishengallis $G$. The inflammophilic character of the periodontitis-associated microbiota. Mol Oral Microbiol 2014; 29: 248-257.

88. Schwarzberg K, Le R, Bharti B et al. The personal human oral microbiome obscures the effects of treatment on periodontal disease. PLoS One 2014; 9: e86708.

89. Forner L, Larsen T, Kilian M, Holmstrup P. Incidence of bacteremia after chewing, tooth brushing and scaling in individuals with periodontal inflammation. J Clin Periodontol 2006; 33: 401-407.

90. Han $Y W$, Wang $X$. Mobile microbiome: oral bacteria in extra-oral infections and inflammation. J Dent Res 2013; 92: 485-491.

91. Arimatsu K, Yamada H, Miyazawa $\mathrm{H}$ et al. Oral pathobiont induces systemic inflammation and metabolic changes associated with alteration of gut microbiota. $\mathrm{SCi}$ Rep 2014; 4: 4828.

92. Chapple I L, Genco R. Diabetes and periodontal diseases: consensus report of the Joint EFP/AAP Workshop on Periodontitis and Systemic Diseases. J Clin Periodontol 2013; 40 Suppl 14: S106-S112.

93. de Pablo P, Chapple I L, Buckley C D, Dietrich T. Periodontitis in systemic rheumatic diseases. Nat Rev Rheumatol 2009; 5: 218-224.

94. Bizzarro S, Laine M L, Buijs M J et al. Microbial profiles at baseline and not the use of antibiotics determine the clinical outcome of the treatment of chronic periodontitis. Sci Rep 2016: 6: 20205.

95. Slatkin M. Epigenetic inheritance and the missing heritability problem. Genetics 2009; 182: 845-850.

96. Bikel S, Valdez-Lara A, Cornejo-Granados F et al. Combining metagenomics, metatranscriptomics and viromics to explore novel microbial interactions: towards a systems-level understanding of human microbiome. Comput Struct Biotechnol I 2015: 13: 390-401.

97. de Soet J.J, Zaura E. Caries: een oplosbare ecologische catastrofe? Quality Practice Mondhygiene 2016; 8: 7-11.

98. Page R C, Kornman K S. The pathogenesis of human periodontitis: an introduction. Periodontol 2000 1997; 14: 9-11. 